\title{
TRANSPORTE DE BORO NO SOLO E SUA ABSORÇÃO POR EUCALIPTO( ${ }^{(1)}$
}

\author{
Edson Marcio Mattiello( ${ }^{(2)}$, Hugo Alberto Ruiz ${ }^{(3)}$, Ivo Ribeiro da \\ Silva $^{(3)}$, Nairam Félix de Barros ${ }^{(3)}$, Julio César Lima Neves ${ }^{(3)} \&$ \\ Maurel Behling ${ }^{(4)}$
}

\begin{abstract}
RESUMO
$O$ mecanismo e a magnitude do transporte de $B$ até as raízes das plantas dependem da umidade e do teor do nutriente no solo. As contribuições do fluxo de massa e da difusão para o transporte de $B$ até as raízes de eucalipto foram avaliadas em resposta ao potencial de água do solo e às doses do nutriente. No ensaio, foram utilizados dois potenciais de água do solo (-10 e -40 $\mathrm{kPa})$ e seis doses de $\mathrm{B}(0,0,5,1$, $2,3 \mathrm{e} 5 \mathrm{mg} \mathrm{dm}^{-3} \mathrm{de} \mathrm{B}$ ). As plantas foram cultivadas em vasos com capacidade de $2,5 \mathrm{dm}^{3} \mathrm{em}$ casa de vegetação. $O$ controle do potencial da água do solo foi efetuado pelo uso de um tensiômetro por vaso, e o ajuste por acréscimo de água destilada. A máxima produção de matéria seca de raízes foi obtida nas doses correspondentes a 0,98 e 2,38 mg dm $\mathrm{mg}^{-3} \mathrm{de}$ nos potenciais de água de -10 e-40 kPa, respectivamente. A máxima produção de matéria seca de parte aérea foi obtida nas doses correspondentes a 0,96 e 1,82 $\mathrm{mg} \mathrm{dm}^{-3}$ de $\mathrm{B}$ nos potenciais de água de -10 e -40 kPa, respectivamente. Foram observadas relações positivas e altamente significativas $(p<0,01)$ entre as doses de B aplicadas, o B extraível do solo, o B na solução do solo e o conteúdo de $\mathrm{B}$ na planta, para ambos os potenciais de água. $O$ fluxo de massa foi o mecanismo predominante no transporte de $B$, chegando a suprir $100 \%$ das necessidades das plantas nas doses mais elevadas do nutriente. $O$ fluxo difusivo foi o mecanismo complementar de maior importância relativa no solo com baixo teor de B e com déficit hídrico.
\end{abstract}

Termos de indexação: fluxo de massa, solução do solo, déficit hídrico, deficiência e toxidez de boro.

\footnotetext{
(1) Parte da Tese de Doutorado do primeiro autor apresentada ao Programa de Pós-Graduação em Solos e Nutrição de Plantas da Universidade Federal de Viçosa - UFV. Apoio Financeiro CNPq e FAPEMIG. Recebido para publicação em junho de 2008 e aprovado em junho de 2009.

(2) Engenheiro-Agrônomo e Doutor em Solos e Nutrição de Plantas, Universidade Federal de Viçosa - UFV. Av. Peter Henry Rolfs s/n, CEP 36571-000 Viçosa (MG). E-mail: mattielloem@yahoo.com.br

(3) Professor do Departamento de Solos, UFV. Bolsista do CNPq. E-mails: hruiz@solos.ufv.br; irsilva@solos.ufv.br; nfbarros@ufv.br; julio_n2003@yahoo.com.br

${ }^{(4)}$ Doutorando do Programa de Pós-Graduação em Solos e Nutrição de Plantas, UFV. E-mail: maurelbehling@yahoo.com.br
} 


\title{
SUMMARY: TRANSPORT OF BORON IN SOIL AND ITS UPTAKE BY EUCALYPT
}

\begin{abstract}
The mechanism and magnitude of $B$ transport to plant roots depend on both water and $B$ soil contents. The contribution of mass flow and diffusion to the B transport in soil to eucalypt roots was evaluated in response to the water potential and $B$ rates. Two water potentials $(-10$ and $-40 \mathrm{kPa})$ and six $B$ levels $\left(0 ; 0.5 ; 1 ; 2 ; 3\right.$ and $5 \mathrm{mg} \mathrm{dm}^{-3}$ of $\left.\mathrm{B}\right)$ were evaluated. The plants were grown in $2.5 \mathrm{dm}^{3}$ plastic pots with soil, in a greenhouse. The water potential was controlled by a tensiometer in each pot and soil moisture adjusted with distilled water. The maximum root dry weight was produced at rates of 0.98 and $2.38 \mathrm{mg} \mathrm{dm}^{-3}$ of $B$, at potentials of -10 and $-40 \mathrm{kPa}$, respectively, and the maximum shoot dry weight at rates of 0.96 and $1.82 \mathrm{mg} \mathrm{dm}^{-3}$ of $B$, at potentials of -10 and $-40 \mathrm{kPa}$, respectively. Positive and highly significant relationships were observed between $B$ rates, soil extractable B, B in soil solution and B plant content at both water potentials. Mass flow was the predominant mechanism for B transport in soil, supplying $100 \%$ of the plant demand in the soils treated with the highest B rates. Diffusion was a complementary mechanism, but its relative increased substantially under conditions of low soil B and greater water deficit.
\end{abstract}

Index terms: Mass flow, soil solution, water deficit, boron deficiency and toxicity.

\section{INTRODUÇÃO}

Fatores ambientais, como deficiências hídricas e nutricionais, têm sido importantes redutores da produção de florestas plantadas no Brasil, pois limitam o crescimento das plantas, com consequente redução de produtividade.

O B é, entre os micronutrientes, aquele que mais frequentemente se apresenta deficiente no solo. No Brasil, solos deficientes em B são encontrados em várias regiões, com destaque para o Cerrado, onde o cultivo do eucalipto tem se expandido. Entre outros autores, Sgarbi et al. (1999) mostraram que a deficiência de B é uma das mais limitantes do crescimento do eucalipto na fase jovem. Um sintoma característico dessa deficiência é a morte do ápice dos ramos, conhecida como seca-de-ponteiro, e a superbrotação lateral. A seca-do-ponteiro vem reduzindo-se com a aplicação de boro em povoamentos de eucalipto. Sgarbi \& Silveira (1999) verificaram redução de $35 \%$ da secade-ponteiro com aplicação de $2,2 \mathrm{~kg} \mathrm{ha}^{-1} \mathrm{de} \mathrm{B}$ em solo arenoso, e de $45 \%$ em solo argiloso na região de TrêsMarias (MG). O problema tem causado prejuízos expressivos, pois afeta a produção e a qualidade da madeira. Além disso, a brotação lateral dificulta a colheita, principalmente em sistemas mecanizados. As quantidades de B aplicadas em povoamentos de eucalipto têm aumentado nos últimos anos, na tentativa de minimizar o problema. No entanto, com frequência, a resposta em produção não tem acompanhado a fertilização com B, mesmo com aplicação de doses mais elevadas.

A baixa eficiência da adubação com $B$ pode estar associada com o período de baixa disponibilidade de água no sistema solo-planta. A falta de água diminui a aquisição de $\mathrm{B}$ pelas raízes das plantas, devido à redução do seu transporte no solo. A transpiração é um fator-chave para a absorção e concentração de B no tecido vegetal (Raven, 1980; Hu \& Brown, 1997). Portanto, a redução no transporte de água pode reduzir o acúmulo de $\mathrm{B}$ em níveis tóxicos no tecido, a exemplo do que ocorre em regiões áridas com solos ricos em $\mathrm{B}$ (Nable et al., 1997), mas também pode limitar o fornecimento de $\mathrm{B}$, promovendo a deficiência do nutriente, como ocorre no Cerrado brasileiro.

A absorção de nutrientes pelas plantas exige seu transporte até a superfície radicular, com destaque para os mecanismos de fluxo de massa (transporte convectivo) e difusão (transporte difusivo) (Nye \& Tinker, 1977; Barber, 1995). Há poucos estudos disponíveis sobre o transporte de B nos solos tropicais. No entanto, há sugestões de que o fluxo de massa é o mecanismo predominante para o transporte do B até as raízes das plantas (Malavolta et al., 1997), estando associado ao gradiente de potencial hídrico total, que regula o movimento da água no sistema solo-plantaatmosfera. Assim, a concentração do íon na solução do solo e a taxa de transpiração do vegetal determinam a quantidade de íons transportada por meio desse mecanismo (Barber, 1995). Matematicamente, o transporte de nutrientes por fluxo de massa é dado pela equação:

$$
\mathrm{FM}=\mathrm{qC}
$$

em que FM: fluxo de massa, $\mathrm{g} \mathrm{cm}^{-2} \mathrm{~s}^{-1}$; : fluxo do fluido, $\mathrm{cm}^{3} \mathrm{~cm}^{-2} \mathrm{~s}^{-1}$; e C: concentração média do soluto na solução, $\mathrm{g} \mathrm{cm}^{-3}$.

A difusão, por sua vez, se deve ao movimento térmico, aleatório, dos íons ou moléculas em meio líquido. $\mathrm{O}$ gradiente de concentração provoca um movimento maior de íons na direção dos pontos de absorção do que em sentido oposto (Wild, 1981). A adaptação das leis de Fick para solos permitiu 
quantificar o transporte difusivo. A primeira lei de Fick pode ser escrita na forma:

$$
\mathrm{F}=-\mathrm{D} \nabla \mathrm{C}
$$

em que F: fluxo do nutriente, $\mathrm{g} \mathrm{cm}^{-2} \mathrm{~s}^{-1}$; D: coeficiente de difusão, $\mathrm{cm}^{2} \mathrm{~s}^{-1} ;$ e $\nabla \mathrm{C}$ : gradiente de concentração, $\mathrm{g} \mathrm{cm}^{-3} \mathrm{~cm}^{-1}$.

O coeficiente de difusão, $\mathrm{D}$, é definido pela equação:

$$
\mathrm{D}=-\mathrm{D}_{1} \theta \mathrm{f} \delta \mathrm{I} / \delta \mathrm{Q}
$$

em que $\mathrm{D}_{1}$ : coeficiente de difusão do elemento, $\mathrm{cm}^{2} \mathrm{~s}^{-1}$; $\theta$ : conteúdo volumétrico de água no solo, $\mathrm{cm}^{3} \mathrm{~cm}^{-3} ; \mathrm{f}$ : fator de impedância, adimensional; $\delta \mathrm{I} / \delta \mathrm{Q}$ : inverso do fator capacidade.

$\mathrm{O}$ sinal negativo indica que o fluxo ocorre do ponto de maior para o de menor concentração. Dadas as dificuldades metodológicas para estimar a difusão usando a lei de Fick, esta tem sido obtida pela diferença entre o conteúdo do nutriente na planta e a quantidade potencialmente transportada por fluxo de massa (Ruiz et al., 1999).

O transporte de B no solo, sua absorção e distribuição na planta são importantes componentes para o entendimento do comportamento desse nutriente no sistema solo-planta. Assim, neste estudo objetivou-se avaliar a contribuição do fluxo de massa e da difusão para o transporte de $\mathrm{B}$ até as raízes de mudas de eucalipto, em resposta ao potencial da água do solo e às doses do nutriente, em ensaio em casa de vegetação com amostras de um Latossolo VermelhoAmarelo distrófico.

\section{MATERIAL E MÉTODOS}

Foi realizado um ensaio em casa de vegetação para avaliar a contribuição do fluxo de massa e da difusão para o transporte de $\mathrm{B}$ no solo até as raízes de eucalipto, em resposta ao potencial da água do solo e às doses do nutriente.

Os tratamentos corresponderam a um arranjo fatorial $2 \times 6$, sendo dois potenciais da água do solo $(-10$ e $-40 \mathrm{kPa}$ ) e seis doses de $\mathrm{B}$, correspondentes a 0 , $0,5,1,2,3$ e $5 \mathrm{mg} \mathrm{dm}^{-3} \mathrm{de} \mathrm{B}$, num delineamento em blocos casualizados, com quatro repetições. No solo estudado, o potencial de água do solo de - $10 \mathrm{kPa}$ referese aproximadamente à capacidade de campo, enquanto o potencial de $-40 \mathrm{kPa}$ corresponde a uma condição de déficit hídrico.

Mudas do clone de Eucaplyptus grandis denominado 129 , selecionado na região de Belo Oriente-MG, foram cultivadas em vasos com capacidade de $2,5 \mathrm{dm}^{3}$, preenchidos com amostras de um Latossolo VermelhoAmarelo distrófico da região de Três Marias, MG (Quadro 1), com teores de água correspondentes aos potenciais de -10 e $-40 \mathrm{kPa}$.

Previamente ao transplantio (uma muda por vaso), o solo foi corrigido incorporando-se $710 \mathrm{mg} \mathrm{dm}^{-3} \mathrm{de}$ calcário dolomítico (PRNT $98 \%$ ), $88 \mathrm{mg} \mathrm{dm}^{-3} \mathrm{de} \mathrm{N} \mathrm{e}$ $200 \mathrm{mg} \mathrm{dm}{ }^{-3}$ de $\mathrm{P}$ (fonte: $\mathrm{NH}_{4} \mathrm{H}_{2} \mathrm{PO}_{4}$ ) e $144 \mathrm{mg} \mathrm{dm}^{-3}$ de $\mathrm{K}$ (fonte: $\mathrm{KCl}$ ). Após o transplantio, as mudas permaneceram por 21 dias com umidade mantida na faixa correspondente a $-10 \mathrm{kPa}$. O controle do potencial de água do solo foi efetuado por meio de um

Quadro 1. Características químicas e físicas do Latossolo Vermelho-Amarelo e da solução do solo extraída em dois potenciais de água do solo $(\Psi)$

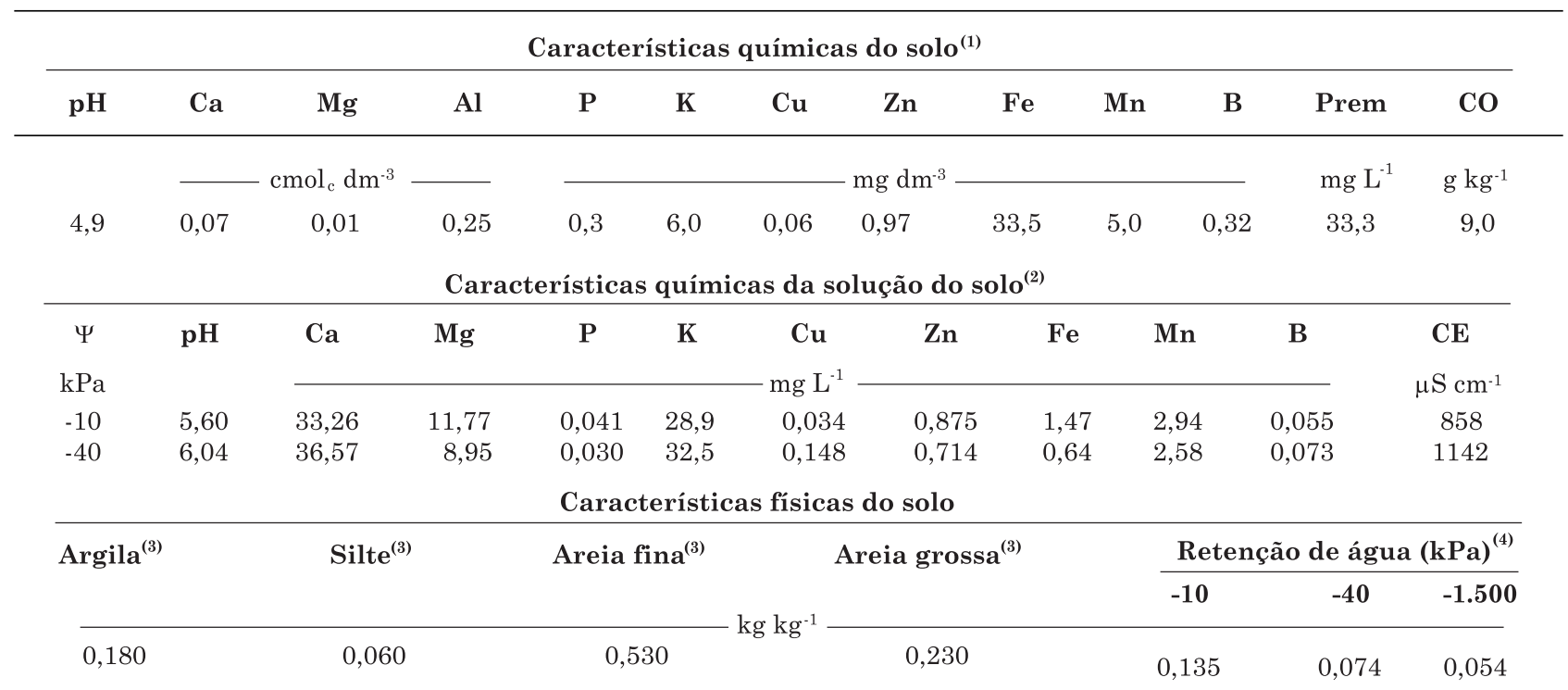

(1) Determinações: pH em água; solo:solução 1:2,5; Ca, $\mathrm{Mg}$ e $\mathrm{Al}$, extrator $\mathrm{KCl} 1 \mathrm{~mol} \mathrm{~L}{ }^{-1}$ (Embrapa, 1997); $\mathrm{P}, \mathrm{K}, \mathrm{Cu}, \mathrm{Zn}, \mathrm{Fe}, \mathrm{Mn}$, extrator Mehlich 1 (Defelipo \& Ribeiro, 1981); B, água quente (Berger \& Truog, 1939); Prem, P remanescente (Alvarez V. et al., 2000); CO, carbono orgânico, método Walkley-Black modificado (Defelipo \& Ribeiro, 1997). ${ }^{(2)} \mathrm{Ca}, \mathrm{Mg}, \mathrm{P}, \mathrm{K}, \mathrm{Cu}, \mathrm{Zn}, \mathrm{Fe}, \mathrm{Mn}$ e $\mathrm{B}$ determinados por ICP-OES. ${ }^{(3)}$ Ruiz (2005). ${ }^{(4)}$ Richards (1949). 
tensiômetro instalado em cada vaso, e o ajuste, por acréscimo de água destilada. Determinações analíticas apontaram a inconveniência da utilização de água deionizada nesses ensaios, pois os teores de $\mathrm{B}$ eram maiores que os encontrados na água destilada, provavelmente pela baixa ionização dos compostos de B e sua baixa afinidade pelas colunas iônicas do deionizador.

Decorrido o período de adaptação, aplicaram-se os tratamentos. As doses de $\mathrm{B}$ foram incorporadas em solução, utilizando $\mathrm{H}_{3} \mathrm{BO}_{3}$ p.a. como fonte. Em acréscimo, metade das unidades experimentais permaneceu com conteúdo de água correspondente a -10 kPa; na outra metade, não houve reposição de água até que se atingisse o potencial de trabalho de $-40 \mathrm{kPa}$. Em cada bloco, dois vasos adicionais com $2,5 \mathrm{dm}^{3} \mathrm{de}$ solo, sem plantas, receberam quantidades de água correspondentes a -10 e a $-40 \mathrm{kPa}$, para quantificar as perdas de água por evaporação.

Durante a realização do experimento, foram feitas três adubações com solução de $\mathrm{NH}_{4} \mathrm{NO}_{3}$ e $\left(\mathrm{NH}_{4}\right)_{2} \mathrm{SO}_{4}$, totalizando aplicação de $120 \mathrm{mg} \mathrm{dm}^{-3} \mathrm{de} \mathrm{Ne} 88 \mathrm{mg} \mathrm{dm}^{-3}$ de $\mathrm{S}$. Os micronutrientes, exceto $\mathrm{B}$, foram também adicionados após o transplantio, em cinco aplicações, totalizando 6,$0 ; 5,5 ; 2,33 ; 2,0$; e $0,23 \mathrm{mg} \mathrm{dm}^{-3} \mathrm{de} \mathrm{Zn}$, $\mathrm{Mn}, \mathrm{Fe}, \mathrm{Cu}$ e Mo, respectivamente. Os micronutrientes foram aplicados na forma de cloreto ou sulfato, excetuando-se o ferro, adicionado como Fe-EDTA.

A quantidade de água acrescentada a cada unidade experimental durante a realização do experimento permitiu calcular a evapotranspiração. A transpiração, no período correspondente, foi obtida por diferença entre a água evapotranspirada na unidade experimental e a água perdida por evaporação nos vasos sem plantas. $\mathrm{O}$ volume de água transpirada foi utilizado nos cálculos para estimativa da contribuição do fluxo de massa.

O ensaio foi encerrado 70 dias após a aplicação dos tratamentos. Foram separadas as diferentes partes da planta, amostrando-se raízes, caules, folhas maduras e folhas jovens (até o terceiro par a partir do ápice dos ramos). O material vegetal foi levado à estufa de circulação de ar forçada por $72 \mathrm{~h}$, a $65^{\circ} \mathrm{C}$, quantificando-se, por meio de pesagem, a produção de matéria seca das diferentes partes. Todo o material foi moído (<5 mm) em moinho tipo Wiley. Amostras de aproximadamente $0,25 \mathrm{~g}$ foram calcinadas por $3 \mathrm{~h}$, a $550{ }^{\circ} \mathrm{C}$, em cadinhos de porcelana. Em seguida, o resíduo da calcinação foi dissolvido em $10 \mathrm{~mL}$ de ácido clorídrico $0,1 \mathrm{~mol} \mathrm{~L}^{-1}$, passado por filtro com poro de $0,45 \mu \mathrm{m}$ e armazenado em recipientes plásticos para análises. Os teores de B no resíduo foram quantificados por meio de espectrofotometria de emissão óptica, com plasma acoplado por indução (ICP-OES).

De cada unidade experimental foram retiradas amostras de solo, cujas soluções foram extraídas com equipamento de membrana de pressão. As amostras de solo receberam água correspondente aos potenciais de trabalho (-10 e - $40 \mathrm{kPa}$ ), permanecendo por $8 \mathrm{~h}$ em sacos plásticos vedados, para atingir o equilíbrio. A solução do solo foi retirada pela aplicação de $1.000 \mathrm{kPa}$ de pressão no sistema, por $16 \mathrm{~h}$, determinando-se, posteriormente, a concentração de B na mesma por meio de ICP-OES. A quantidade de B transportada por fluxo de massa $\left(\mathrm{Q}_{\mathrm{FM}}\right)$ foi calculada com base na concentração de B na solução do solo (C) multiplicada pelo volume de água transpirada no período experimental (V), no intervalo de 21-91 dias, da seguinte forma:

$$
\begin{array}{ll}
\text { Para }-10 \mathrm{kPa}: & \mathrm{Q}_{\mathrm{FM}}=\mathrm{C}_{-10 \mathrm{kPa}} \mathrm{V}_{21-91} \\
\text { Para }-40 \mathrm{kPa}: & \mathrm{Q}_{\mathrm{FM}}=\mathrm{C}_{-40 \mathrm{kPa}} \mathrm{V}_{21-91}
\end{array}
$$

$\mathrm{O}$ acúmulo de $\mathrm{B}$ na planta $\left(\mathrm{Q}_{\mathrm{P}}\right)$, no intervalo de 2191 dias, foi obtido pela diferença entre o conteúdo total de $B\left(Q_{T}\right)$, o acúmulo no período de adaptação $\left(Q_{A}\right)$, no intervalo de $0-20$ dias, e a quantidade inicial de $\mathrm{B}$ nas mudas transplantadas $\left(\mathrm{Q}_{\mathrm{i}}\right)$ :

$$
\left(\mathrm{Q}_{\mathrm{P}}\right)=\mathrm{Q}_{\mathrm{T}}-\mathrm{Q}_{\mathrm{A}^{-}}-\mathrm{Q}_{\mathrm{i}}
$$

A contribuição da difusão $\left(\mathrm{Q}_{\mathrm{D}}\right)$ foi calculada pela equação:

$$
\mathrm{Q}_{\mathrm{D}}=\mathrm{Q}_{\mathrm{P}}-\mathrm{Q}_{\mathrm{FM}}
$$

Nas amostras de solo, determinou-se também o B extraído em água quente (Berger \& Truog, 1939). Assim, consideraram-se neste trabalho as doses de B aplicadas (B), o B extraído em água quente (B solo) e a concentração de B na solução do solo (B solução).

Os resultados experimentais foram analisados estatisticamente, comparando o potencial da água do solo pelo teste $\mathrm{F}$ e as doses de $\mathrm{B}$, dentro de cada potencial, por regressão.

\section{RESULTADOS E DISCUSSÃO}

\section{Aspectos visuais}

No decorrer do ensaio, nas doses elevadas de B (3 e $5 \mathrm{mg} \mathrm{dm}^{-3} \mathrm{de}$ B) e no potencial de água de $-10 \mathrm{kPa}$, foram observados sintomas visuais de toxidez de $\mathrm{B}$, a partir dos 20 dias após aplicação dos tratamentos (Figura 1a, b). As plantas submetidas ao potencial de água de $-40 \mathrm{kPa}$ apresentaram sintomas de toxidez mais tardiamente, apenas na dose de $5 \mathrm{mg} \mathrm{dm}^{-3} \mathrm{de} \mathrm{B}$. Os sintomas de toxidez de B manifestaram-se em folhas maduras, com clorose nas bordas foliares e, posteriormente, com manchas necróticas. Houve queda dessas folhas, que apresentaram teores de $1.350 \mathrm{mg} \mathrm{kg}^{-1} \mathrm{de}$ B. Os sintomas de toxidez coincidem com regiões das folhas onde há maior transpiração (Nable et al., 1997).

Por outro lado, 35 dias após aplicação dos tratamentos, foram observados sintomas típicos de deficiência de B nas plantas testemunhas, com 

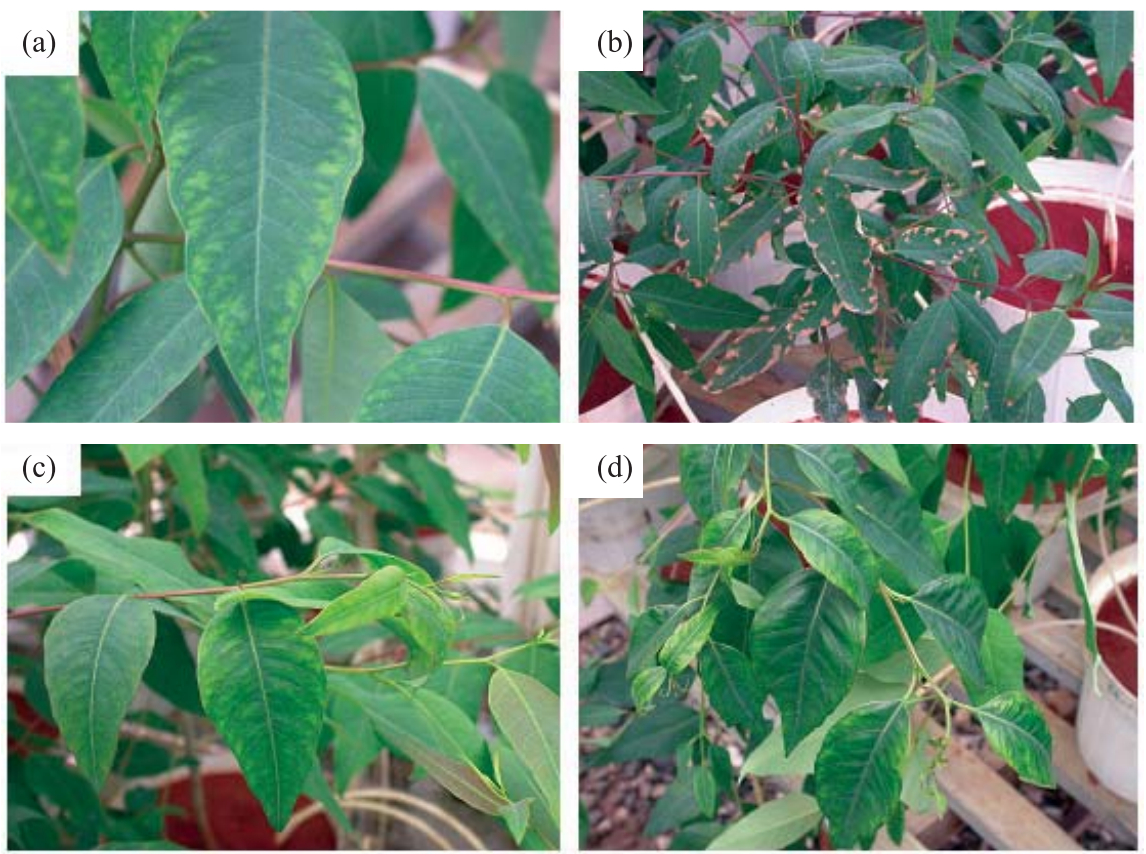

Figura 1. Estádio inicial e mais avançado de toxidez e deficiência de B em eucalipto. (a) toxidez inicial; (b) toxidez avançada; (c) deficiência inicial; e (d) deficiência mais avançada.

expressão mais severa nas plantas crescidas a $-40 \mathrm{kPa}$ (Figuras 1c, d). Algumas plantas apresentaram clorose nas folhas jovens, seguida de encarquilhamento, que tornou essas folhas quebradiças. Acentuada a deficiência, verificou-se queda de folhas e morte da gema apical do caule em algumas plantas.

\section{Produção de matéria seca}

A maior disponibilidade de água proporcionou maior produção de matéria seca, independentemente da dose de $\mathrm{B}$ (Figura 2). O incremento médio na produção de matéria seca de raízes e de parte aérea foi de 120 e 110 \%, respectivamente. A produção de matéria seca de raízes e parte aérea foi influenciada pelas doses de B e pelo potencial de água do solo. Não obstante, o ajuste das médias aos modelos raiz quadrática e quadrático, com o ponto de máxima produção dentro do intervalo experimental, indica resposta negativa às maiores doses de $\mathrm{B}$ aplicadas no solo. O ajuste do melhor modelo encontrado (raiz quadrada) aos dados de produção de matéria seca, no potencial de água de $-10 \mathrm{kPa}$, não permite a estimativa do nível crítico para B, aqui definido como $90 \%$ da produção máxima, uma vez que os teores críticos seriam muito baixos e irreais. Portanto, a discussão e as estimativas posteriores serão feitas com base na máxima eficiência física.

A máxima produção de matéria seca de raízes foi obtida nas doses correspondentes a 0,98 e $2,38 \mathrm{mg} \mathrm{dm}^{-3}$ de $\mathrm{B}$, nos potenciais de água de -10 e $-40 \mathrm{kPa}$, respectivamente. A máxima produção de matéria seca de parte aérea foi obtida nas doses correspondentes a 0,96 e 1,82 $\mathrm{mg} \mathrm{dm}^{-3}$ de B, nos potenciais de água de

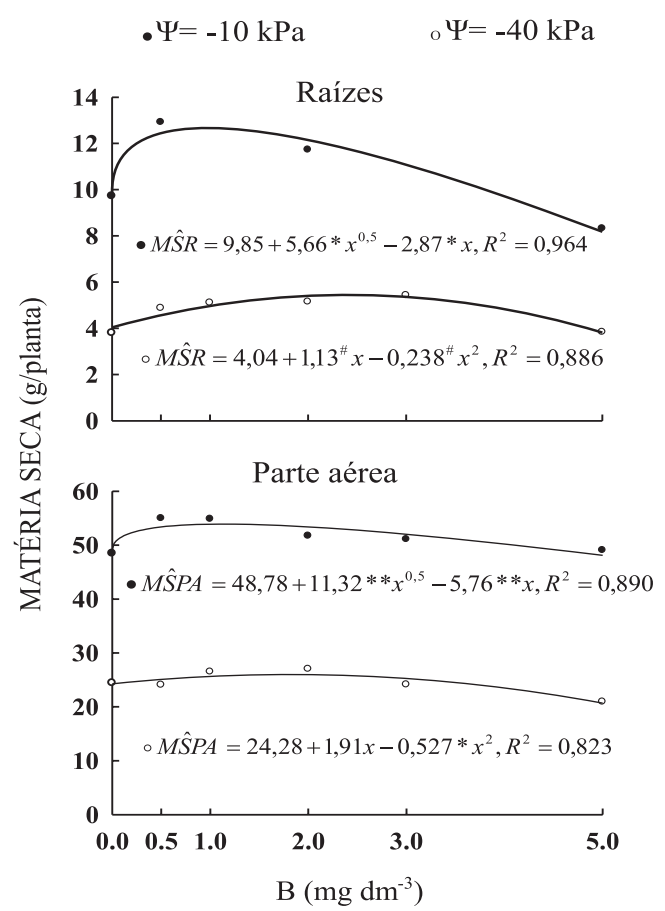

Figura 2. Produção de matéria seca de raízes (MSR) e de parte aérea (MSPA) do eucalipto em resposta às doses de $\mathrm{B}$, considerando o potencial de água do solo $(\Psi) .{ }^{\#}, * \mathrm{e} * *$ significativo a $10,5 \mathrm{e}$ $1 \%$, respectivamente, pelo teste $t$.

-10 e $-40 \mathrm{kPa}$, respectivamente. Os resultados mostram que a eficiência da adubação com B é maior quando há maior disponibilidade de água no solo, sendo necessárias doses mais elevadas para atingir a 
máxima eficiência física sob déficit hídrico; contudo, a produção obtida será menor. Viets (1972) ressalta que, com estresse hídrico, os teores de B no solo têm efeito relativo, mascarado pela sua menor disponibilidade, visto que nas mesmas doses houve aumento na produção de matéria seca pela maior disponibilidade de água.

Na comparação entre a dose zero e a dose de máxima eficiência física, houve incremento de $22 \mathrm{e}$ $25 \%$ na produção de matéria seca de raízes, nos potenciais de água de -10 e $-40 \mathrm{kPa}$, respectivamente. $\mathrm{O}$ incremento na produção de matéria seca de parte aérea foi de 11 e 9,5\% nos potenciais de água de - 10 e $-40 \mathrm{kPa}$, respectivamente. É sabido que as raízes são mais sensíveis à deficiência de $\mathrm{B}$ do que a parte aérea (Gupta et al., 1985; Shelp, 1993; Marschner, 1995; Dell \& Huang, 1997). Lehto (1994) e Mottonen et al. (2001b) verificaram redução do número de raízes finas e micorrizas em plantas deficientes em $\mathrm{B}$, fato que pode estar relacionado com a limitada absorção de água. Adicionalmente, a deficiência de $\mathrm{B}$ reduz a permeabilidade da membrana plasmática e o fluxo de água, alterando a absorção dos nutrientes. A redução da condutância hidráulica do xilema pode estar associada à menor transpiração, à menor absorção de $\mathrm{B}$ e ao crescimento de plantas sob deficiência de B (Nable et al., 1997; Dannel et al., 1998). O papel estrutural do $\mathrm{B}$ e aqueles associados às funções de divisão, diferenciação e expansão celular devem estar associados com a alteração do potencial hidráulico da planta (Nable et al., 1997; Dannel et al., 1998).

\section{Transporte de B no solo}

$\mathrm{Na}$ figura 3 é apresentada a relação entre o B extraído do solo e o B na solução do solo em resposta à dose deste nutriente nos dois potenciais de água. $\mathrm{O}$ comportamento do B no solo foi linear positivo em função da dose aplicada, enquanto o B na solução do solo teve comportamento quadrático, evidenciando ligeiro tamponamento do solo para o B. Considerando a máxima produção de parte aérea, pode-se estimar $\mathrm{B}$ no solo e o B na solução do solo para obtenção da máxima eficiência física. Assim, no potencial de água de $-10 \mathrm{kPa}$, a máxima produção de parte aérea foi obtida com teores de B no solo de $0,33 \mathrm{mg} \mathrm{dm}^{-3}$ e B na solução de $0,101 \mathrm{mg} \mathrm{L}^{-1}$. Sob déficit hídrico, a máxima eficiência física foi obtida em valores superiores de $\mathrm{B}$ no solo $\left(0,738 \mathrm{mg} \mathrm{dm}^{-3}\right)$ e B na solução $\left(0,308 \mathrm{mg} \mathrm{L}^{-1}\right)$. O estresse hídrico diminui a absorção e a distribuição do B na planta, aumentando o requerimento externo do nutriente (Dell \& Huang, 1997).

Os valores obtidos para fluxo de massa e conteúdo de $\mathrm{B}$ na planta foram ajustados às doses de $\mathrm{B}$, ao $\mathrm{B}$ extraído do solo e ao B na solução, para cada potencial de água do solo, por meio de regressão (Figura 4). Uma vez que a difusão complementa o transporte por fluxo de massa, sua contribuição foi obtida pela diferença entre o conteúdo de B na planta e a quantidade potencialmente transportada por fluxo de massa.
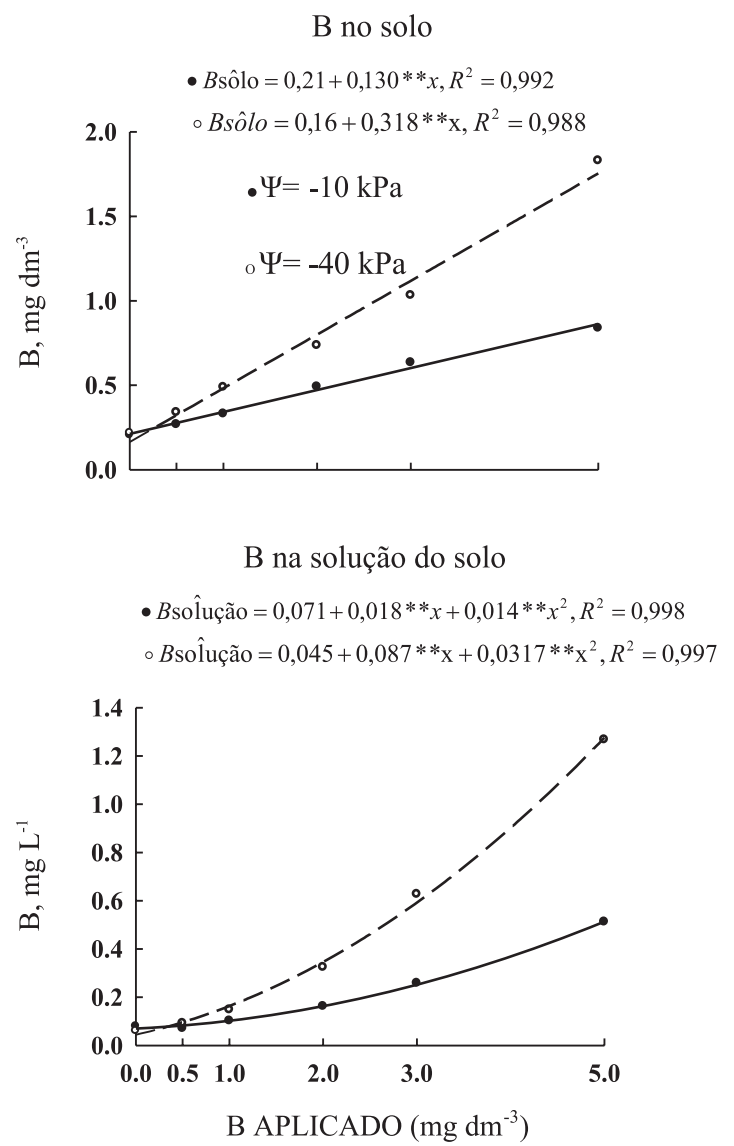

Figura 3. Teores de B no solo e B na solução do solo em resposta às doses de $B$ ( $B$ aplicado), considerando o potencial de água do solo $(\Psi)$. *** significativo a $1 \%$ pelo teste $t$.

Assim, a área compreendida entre as duas curvas é considerada a contribuição por difusão, e o ponto de coincidência entre as duas curvas representa a condição em que o fluxo de massa supre potencialmente toda a demanda da planta (Figura 4).

A contribuição potencial por fluxo de massa e o conteúdo de $\mathrm{B}$ foram influenciados pelo potencial de água do solo e pelas doses de B. As plantas cultivadas no potencial de água de $-10 \mathrm{kPa}$ apresentaram, em média, transpiração 3,5 vezes superior à das plantas cultivadas sob déficit hídrico. A solução do solo correspondente a $-10 \mathrm{kPa}$ apresentou metade da concentração de $\mathrm{B}$ referente àquela $\mathrm{a}-40 \mathrm{kPa}$. Esses valores são devido à diluição do nutriente no maior volume de água do solo e à maior absorção de $\mathrm{B}$ pelas plantas nessa condição de cultivo. Portanto, a maior contribuição do fluxo de massa observada no potencial de água do solo de $-10 \mathrm{kPa}$ está relacionada com a maior transpiração das plantas. Em média, as plantas cultivadas no potencial de água de $-10 \mathrm{kPa}$ acumularam o dobro de $\mathrm{B}$, quando comparadas às plantas cultivadas no potencial de $-40 \mathrm{kPa}$ (Figura 4).

Os resultados mostram que o fluxo de massa é o principal mecanismo de transporte de $\mathrm{B}$ até a 


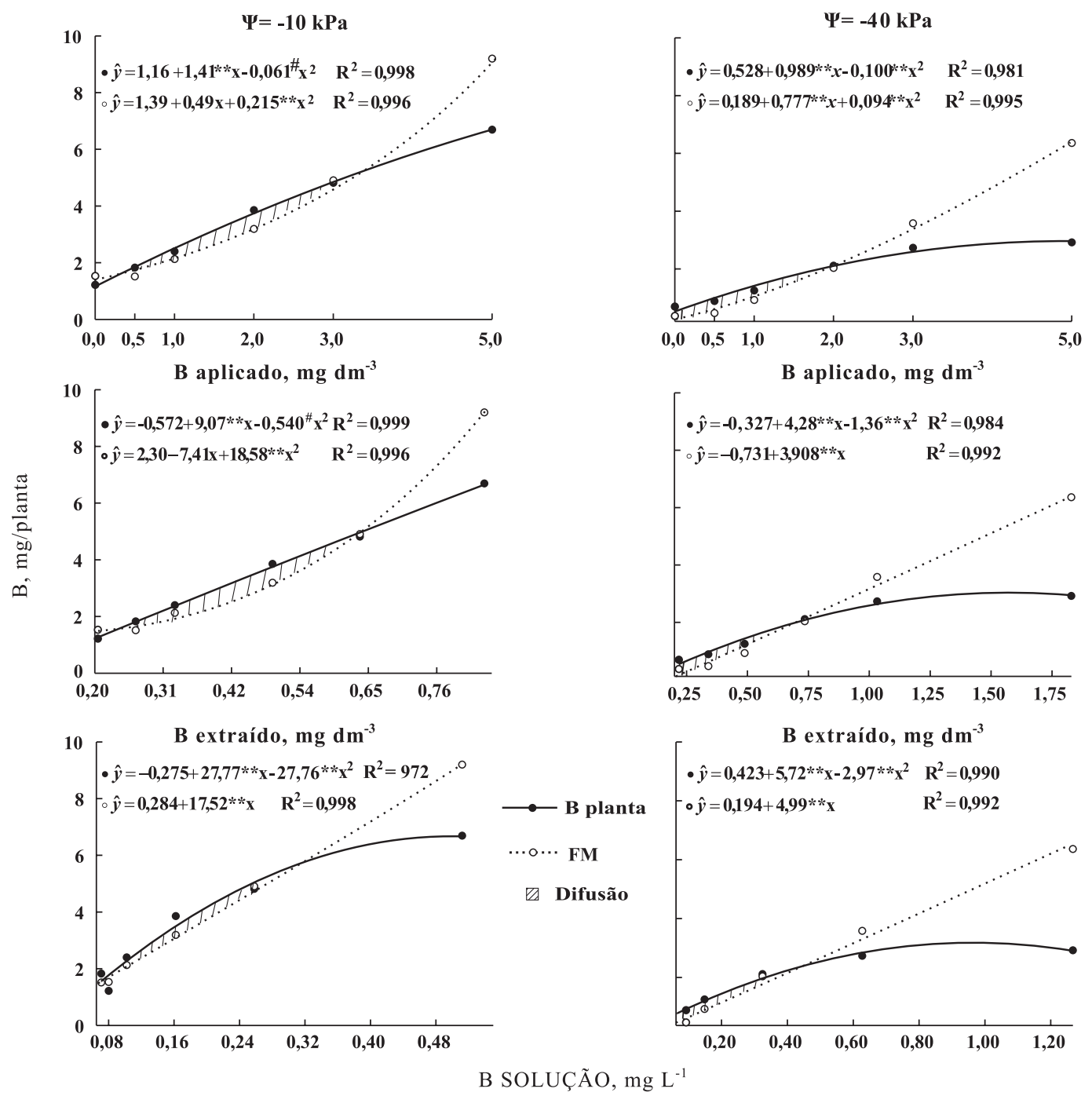

Figura 4. Conteúdo de B na planta (B planta) e B potencialmente transportado por fluxo de massa (FM) em resposta às doses de $\mathrm{B}$ ( $\mathrm{B}$ aplicado), ao teor de $\mathrm{B}$ no solo ( $\mathrm{B}$ extraído) e $\mathrm{B}$ na solução do solo (B solução), considerando o potencial de água do solo ( $\Psi)$. A superfície hachurada representa o B transportado por difusão. ${ }^{\#} \mathrm{e} * *$ significativo a 10 e $1 \%$, respectivamente, pelo teste $\mathrm{t}$.

superfície radicular. Na condição de maior disponibilidade de água $(-10 \mathrm{kPa})$, o fluxo de massa supriu $100 \%$ da demanda de B a partir de valores de dose de 3,0 mg dm ${ }^{-3}$ de B, B no solo de 0,63 $\mathrm{mg} \mathrm{dm}^{-3} \mathrm{e}$ $\mathrm{B}$ na solução de $0,30 \mathrm{mg} \mathrm{L}^{-1}$ (Figura 4). Na condição de déficit hídrico $(-40 \mathrm{kPa})$, o fluxo de massa supriu $100 \%$ da demanda de B a partir de valores de dose de $1,9 \mathrm{mg} \mathrm{dm}^{-3}$ de B, B no solo de $0,69 \mathrm{mg} \mathrm{dm}^{-3}$ e B na solução de $0,43 \mathrm{mg} \mathrm{L}^{-1}$. A difusão mostrou-se importante para o suprimento de B para as plantas, principalmente com déficit hídrico e baixos teores de B no solo. Em solos muito intemperizados, pobres em $\mathrm{B}$ e em regiões onde ocorrem períodos prolongados de seca, a difusão pode ser um mecanismo importante para o suprimento de B para o eucalipto. O fluxo de massa tem importância relativa maior para o suprimento de $\mathrm{B}$ à medida que se elevam os teores deste nutriente no solo e a disponibilidade de água.
Os percentuais de fluxo de massa para $\mathrm{B}$ mostram que, na dose zero de $\mathrm{B}$ e potencial de água de $-10 \mathrm{kPa}$, o fluxo de massa supriu potencialmente $100 \%$ da demanda de $\mathrm{B}$ das plantas, enquanto sob déficit hídrico esse percentual foi de apenas $53 \%$ (Figura 5). As plantas cultivadas em ambos os potenciais de água apresentaram sintomas de deficiência de $\mathrm{B}$, porém a deficiência foi mais severa no potencial de $-40 \mathrm{kPa}$.

A falta de água alterou a contribuição dos mecanismos de transporte de B no solo, sobretudo nas menores doses de B, deprimindo o fluxo de massa e a quantidade de B absorvido pelas plantas. Esses resultados mostram a importância da transpiração das plantas para absorção do B e corroboram as observações de campo em que sintomas de deficiência de B surgem em períodos secos do ano e praticamente desaparecem em períodos chuvosos. Segundo Adriano 


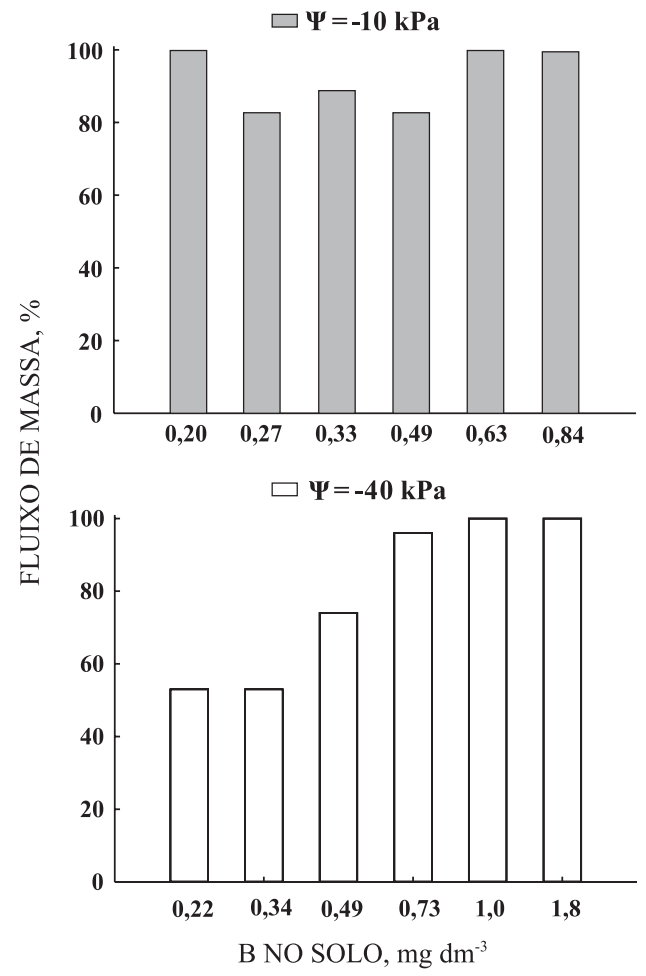

Figura 5. Percentual de $B$ potencialmente transportado por fluxo de massa em resposta ao $B$ extraído do solo (B solo), considerando o potencial de água do solo $(\Psi)$.

(1986) e Loué (1993), a perda de água por meio da transpiração causa um fluxo de massa de íons na superfície das raízes, e a ausência de água pode causar redução expressiva na absorção desses íons pelas plantas.

\section{Teores de B no solo e no tecido vegetal}

De modo geral, os teores de $\mathrm{B}$ foram maiores nas plantas cultivadas em menor disponibilidade de água: -40 kPa (Figura 6). Em relação ao B solo, pode-se verificar aumento progressivo nos teores nos tecidos vegetais à medida que se incrementam os teores no solo (Figura 6). Considerando os teores de B no solo para obtenção da máxima produção de matéria seca de parte aérea, $0,330 \mathrm{mg} \mathrm{dm}^{-3} \mathrm{a}-10$ e $0,738 \mathrm{mg} \mathrm{dm}^{-3}$ a -40 kPa, verifica-se que os teores foliares foram bem superiores nas plantas cultivadas sob déficit hídrico e corresponderam a $112 \mathrm{mg} \mathrm{kg}^{-1}$ de B. As plantas cultivadas a - $10 \mathrm{kPa}$ atingiram máxima produção de matéria seca de parte aérea com teores foliares de $60 \mathrm{mg} \mathrm{kg}{ }^{-1}$ de B. A variação dos teores de B nas raízes e no caule foi menor, porém sempre com valores superiores aos das plantas cultivadas sob déficit hídrico. Nas raízes, esses teores foram de 47 e $28 \mathrm{mg} \mathrm{kg}^{-1}$ de B nos potenciais de -10 e $-40 \mathrm{kPa}$ e, nos caules, de 26 e $21 \mathrm{mg} \mathrm{kg}^{-1}$ de $\mathrm{B}$, respectivamente (Figura 6). Mesmo com teores superiores de B no tecido vegetal, as plantas cultivadas a $-40 \mathrm{kPa}$ não alcançaram a produção de matéria seca das cultivadas a $-10 \mathrm{kPa}$. O menor crescimento das plantas sob déficit hídrico, como verificado na figura 2 , promove maior concentração dos nutrientes no tecido vegetal, fato conhecido como efeito de concentração. Ao contrário, em condições adequadas de umidade do solo (-10 kPa), o crescimento das plantas foi favorecido, ocorrendo diluição dos nutrientes na matéria seca produzida (Jarrel \& Beverry, 1981). Higashi et al. (2000) consideram teores foliares de B entre 35 e $70 \mathrm{mg} \mathrm{kg}^{-1}$ adequados para clones de eucaliptos em mini e microjardins, e valores inferiores a $20 \mathrm{mg} \mathrm{kg}^{-1} \mathrm{e}$ superiores a $70 \mathrm{mg} \mathrm{kg}^{-1}$ constituem os limites para deficiência e toxidez, respectivamente. Malavolta et al. (1997) classificam como adequado teores de B entre 40 e $50 \mathrm{mg} \mathrm{kg}^{-1}$ e deficiente entre 15 e $20 \mathrm{mg} \mathrm{kg}^{-1}$ para Eucalyptus spp. Dell et al. (1995) consideram adequados teores de B entre 13 e $30 \mathrm{mg} \mathrm{kg}^{-1} \mathrm{e}$ deficiente entre 8 e $12 \mathrm{mg} \mathrm{kg}^{-1}$ para $E$. grandis $x$ E. urophylla. Os teores foliares de B podem ser bastante variáveis dependendo do material genético,

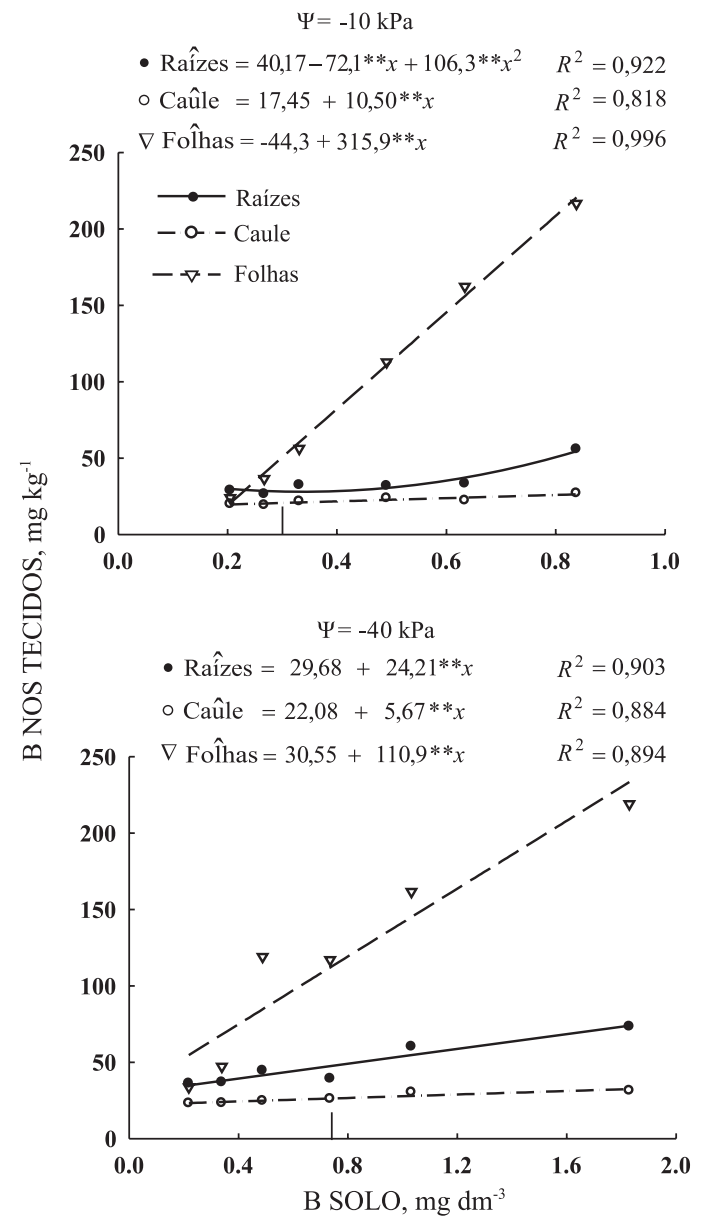

Figura 6. Teores de $B$ nas raízes, caules e folhas do eucalipto em resposta ao teor de B no solo (B solo), considerando o potencial de água do solo ( $\Psi)$. O traço (l) corresponde ao B solo para obtenção da máxima produção de matéria de parte aérea.** significativo a $1 \%$ pelo teste $t$. 
do ambiente de crescimento e da idade das plantas. Neste estudo, os resultados indicam que os teores foliares de B nas plantas de eucalipto variaram de acordo com o regime hídrico e que, em épocas chuvosas, menores teores de B podem ser reflexo do maior crescimento no período. Assim, a comparação de teores foliares de B com seus níveis críticos deve considerar também as condições hídricas do momento da coleta, pois teores menores de $B$ não necessariamente indicam deficiência potencial. Por outro lado, no próprio estabelecimento dos níveis críticos foliares de B devem ser consideradas, entre outras, as condições hídricas e de crescimento da planta, de modo que a avaliação nutricional seja a mais precisa possível.

\section{CONCLUSÕES}

1. O fluxo de massa é o mecanismo predominante para o transporte de B no solo. A difusão é um mecanismo complementar para o transporte de B, de importância relativa maior em solos pobres desse nutriente e em períodos de déficit hídrico.

2. O déficit hídrico altera o balanço dos mecanismos de transporte de B no solo, principalmente nos solos pobres desse nutriente, reduzindo o fluxo de massa e o acúmulo de B na planta.

3. O déficit hídrico eleva os requerimentos externos de $\mathrm{B}$ e promove maior concentração do nutriente no tecido vegetal.

\section{LITERATURA CITADA}

ADRIANO, D.C. Trace elements in the terrestrial environment. New York, Springer Verlag, 1986. 533p.

ALVAREZ V., V.H.; NOVAIS, R.F.; DIAS, L.E. \& OLIVEIRA, J.A. Determinações e uso do fósforo remanescente. B. Inf. SBCS, 25:27-33, 2000.

BARBER, S.A. Soil nutrient bioavailability: A mechanistic approach. 2.ed. New York, John Wiley \& Sons, 1995. p.330-338.

BERGER, K.C. \& TRUOG, E. Boron determination in soils and plants using the quinalizarin reaction. Indus. Eng. Chem. Anal., 11:540-545, 1939.

DANNEL, F.; PFEFFER, H. \& RÖMHELD, V. Compartmentation of boron in roots and leaves of sunflower as affected by boron supply. J. Plant Physiol., 153:615-622, 1998.

DEFELIPO, B.V. \& RIBEIRO, A.C. Análise química de solo. Viçosa, MG, Universidade Federal de Viçosa, 1981. 17p. (Boletim de Extensão)

DEFELIPO, B.V. \& RIBEIRO, A.C. Análise química de solo. Viçosa, MG, Universidade Federal de Viçosa, 1997. 26p. (Boletim de Extensão)
DELL, B. \& HUANG, L. Physiological responses of plants to low boron. Plant Soil, 193:85-101, 1997.

DELL, B.; MALAJCZUK, N. \& GROVE, T.S. Nutrient disorders in plantation eucalypts. Canberra, Australian Center for International Agriculture Research, 1995. 104p.

EMPRESA BRASILEIRA DE PESQUISA AGROPECUÁRIA EMBRAPA. Centro Nacional de Pesquisas de Solos. Manual de análise de solo. 2.ed. Rio de Janeiro, 1997. 212p.

GUPTA, U.C.; JAME, Y.W. \& CAMPBELL, C.A. Boron toxicity and deficiency: A review. Canad. J. Soil Sci., 65:381-409, 1985.

HIGASHI, E.N.; SILVEIRA, R.L.V.A. \& GONCALVES, A.N Propagação vegetativa de Eucalyptus: Princípios básicos e a sua evolução no Brasil. Circular Técnica IPEF, 192:1$11,2000$.

HU, H. \& BROWN, P.H. Absorption of boron by plants. Plant Soil, 193:49-58, 1997.

JARREL, W.M. \& BEVERY, R.B. The dilution effect in plant nutrition studies. Adv. Agron., 34:197-224, 1981.

LEHTO, T. Effects of liming and boron fertilization on mycorrhizae of Picea abies. Plant Soil, 163:65-68, 1994.

LOUÉ, A. Oligoéléments en agricultures. Antibes, SCPANATHAN, 1993. 577p.

MALAVOLTA, E.; VITTI, G.C. \& OLIVEIRA, S.A. Avaliação do estado nutricional das plantas: Princípios e aplicações. 2.ed. Piracicaba, Potafos, 1997. 319p.

MARSCHNER, H. Mineral nutrition of higher plants. 2.ed. London, Academic Press, 1995. p.379-396.

MOTTONEN, M.; LEHTO, T. \& APHALO, P.J. Growth dynamics and mycorrhizas of Norway spruce (Picea abies) seedlings in relation to boron supply. Trees, 15:319-326, $2001 b$.

NABLE, R.O.; BAÑUELOS, G.S. \& PAULL, J.G. Boron toxicity. Plant Soil, 198:181-198, 1997.

NYE, P.H. \& TINKER, P.B. Solute movement in the soil-root system. Berkeley, University of California Press, 1977. $342 \mathrm{p}$.

RAVEN, J.A. Short- and long-distance transport of boric acid in plants. New Phytol., 84:231-249, 1980.

RICHARDS, L.A. Methods of measuring soil moisture tension. Soil Sci., 68:95-112, 1949.

RUIZ, H.A. Incremento da exatidão da análise granulométrica do solo por meio da coleta da suspensão (silte + argila). R. Bras. Ci. Solo, 29:297-300, 2005.

RUIZ, H.A.; MIRANDA, J. \& CONCEIÇÃO, J.C.S. Contribuição dos mecanismos de fluxo de massa e difusão para o suprimento de $\mathrm{K}, \mathrm{Ca}$ e $\mathrm{Mg}$ a plantas de arroz. R. Bras. Ci. Solo, 23:1015-1018, 1999.

SGARBI, F.; SILVEIRA, R.L.V.A.; TAKAHASHI, E.N. \& CAMARGO, M.A.F. Crescimento e produção de biomassa de clone de Eucalyptus grandis x Eucalyptus urophylla em condições de deficiência de macronutrientes, B e $\mathrm{Zn}$. Sci. Flor., 56:69-83, 1999. 
SGARBI, F. \& SILVEIRA, R.L.V.A. Resposta do Eucalyptus à aplicação de boro e potássio na região de Três Marias MG. In: SIMPÓSIO SOBRE O USO DO BORO EM FLORESTAS DE EUCALIPTO, 2., Piracicaba, 1998. Anais. Piracicaba, IPEF/ESALQ, 1999. p.89-105.

SHELP, B.J. Physiology and biochemistry of boron in plants. In: GUPTA, U.C., ed. Boron and its role in crop production. Boca Raton, CRC Press, 1993. p.53-85.
VIETS, F.G. Water deficits and nutrients availability. In: KOZLOWSKI, T.T., ed. Water deficits and plant growth. New York, Academic Press, 1972. p.217-239.

WILD, A. Mass flow and diffusion. In: GRENLAND, D.J. \& HAYES, M.H.B., eds. The chemistry of soil processes. Chichester, John Wiley, 1981. p.37-80. 